\title{
Palliative Care - Hintergründe, Ansätze und Herausforderungen
}

\author{
Elke STEUDTER' \\ Kaleidos Fachhochschule, Departement Gesundheit
}

\begin{abstract}
Der folgende Beitrag führt im Überblick in die Palliative Care ein und zeigt die zentralen Konzepte der auf die Lebensqualität von schwerkranken und sterbenden Menschen fokussierten Ausrichtung innerhalb des Gesundheits-, Pflege- und Sozialwesens. Die Ausführungen basieren auf der Vorlesung «Palliative Care - Möglichkeiten und Grenzen» im Rahmen der Veranstaltungsreihe «Sterben und Tod», die im Frühjahr 2016 im Departement für Sozialwissenschaften an der Universität Fribourg durchgeführt wurde.

Die Ausführungen stellen neben den historischen und gesellschaftlichen Hintergründen die Prinzipien der palliativen Versorgung in den Vordergrund und beleuchten, welche Ansätze darin verfolgt werden und welche Herausforderungen damit verbunden sind.
\end{abstract}

Keywords: Palliative Care; Pflege; Prinzipien; Ansätze

\section{Einleitung}

Die Auseinandersetzung mit dem Sterben und dem Tod gehört zu den wichtigen Aufgaben des Menschen. In früheren Jahrhunderten durch ihre dauernde Präsenz noch fester Bestandteil der Gesellschaft, wurden die Themen mehr und mehr an den Rand des kollektiven und individuellen Bewusstseins gedrängt. Nun setzt eine vor Jahrzehnten begonnene Gegenbewegung ein und die Thematik wird (wieder) Bestandteil des gesellschaftlichen Diskurses. Dabei scheint mehr die menschliche Angst im Zusammenhang mit dem individuellen Sterbeprozess als die unabänderliche Tatsache des Todes im Zentrum der Diskussion zu stehen (Student/Mühlum/Student 2016).

Die gesellschaftliche und sozialwissenschaftliche Auseinandersetzung mit dem Thema konzentriert sich dabei häufig ausschliesslich auf das Lebensende - auf eine Zeit, die lediglich am Schluss eines Kontinuums steht. Damit wird weitgehend vernachlässigt, dass der irdischen Endgültigkeit häufig mehrere Jahre gestaltbaren Lebens vorausgehen, in denen Menschen an einer potenziell lebensbedrohlichen oder chronisch fortschreitenden Krankheit leiden und dabei begleitet bzw. unterstützt werden müssen (BAG/GDK 2009). Daraus leiten sich weitreichende Aufgaben für die Gesellschaft und insbesondere für das Gesundheits-, Pflege- und

\footnotetext{
${ }^{1}$ Elke Steudter, Pflegewissenschaftlerin, Studiengangsleitung MAS FH Geriatric Care, MAS FH Palliative Care, Kalaidos Fachhochschule Gesundheit, Zürich. elke.steudter@kalaidos-fh.ch
} 
Sozialwesen ab. Hier nun setzt die Palliative Care als interprofessionell ausgerichtete Versorgungseinheit an. Abgeleitet vom Lateinischen «pallium» prägt symbolisch der Mantel bzw. die Ummantelung des von Leid betroffenen Menschen das Verständnis dieses auf die Lebensqualität - und nicht auf die Heilung - ausgerichteten Ansatzes (Twycross 2003).

Im Rahmen der im Frühjahr 2016 initiierten Vorlesungsreihe «Sterben und Tod» im Departement für Sozialwissenschaften an der Universität Fribourg wurde für Studierende der Soziologie, Sozialpolitik und Sozialarbeit das Thema Palliative Care aufbereitet und so die Sicht auf das Thema der Veranstaltung um einen wichtigen Aspekt ergänzt. Die folgenden Ausführungen nehmen die Bestandteile der Vorlesung auf und stellen den Ansatz der Palliative Care zunächst vor dem Hintergrund seiner historischen Entwicklung im Kontext der demografischen und epidemiologischen Veränderungen dar. Der geschichtliche Abriss zeichnet die Entstehung auf internationaler und nationaler Ebene nach und beschreibt die Ursprünge des palliativen Denkens. Davon wird die aktuelle Notwendigkeit der palliativen Versorgung abgeleitet und ein grundsätzliches Verständnis von Palliative Care dargelegt. Im Anschluss daran wird anhand der Nationalen Strategie Palliative Care gezeigt, wie der Versorgungsansatz gesellschaftlich und gesundheitspolitisch in der Schweiz eingebettet und verankert ist. Um das Verständnis der Palliative Care in den Sozialwissenschaften weiter zu vertiefen, werden die zentralen Anliegen und Konzepte beschrieben und diese unter den Aspekten ihrer Möglichkeiten und Herausforderungen beleuchtet.

\section{Entstehung und historischer Kontext}

Auch wenn die Entwicklung und Etablierung des Palliative Care Ansatzes eng mit dem Leben und Wirken der Engländerin Dame Cicely Saunders verbunden ist, so nimmt die professionelle Versorgung von schwerkranken und sterbenden Menschen ihren Anfang in den USA und in Irland. Dort werden bereits seit Ende des 19. Jahrhunderts Menschen, die hauptsächlich an weit fortgeschrittenen Tumorerkrankungen leiden, in christlich geprägten Hospizen gepflegt (Buck 2007). Zu Beginn des 20. Jahrhunderts verbreitet sich dieser Ansatz immer mehr und in verschiedenen Ländern entstehen weitere Einrichtungen.

In den 1950er Jahren wird der Bedarf für eine neue Versorgung von schwerkranken und sterbenden Menschen auch im Vereinten Königreich immer deutlicher. In mehreren national angelegten Untersuchungen zeigte sich, dass Menschen mit Tumorerkrankungen in der letzten Lebensphase grossem Leid ausgesetzt sind und dass ihre Behandlung und Pflege nur unzureichend gewährleistet werden kann (Saunders 2001). In der Folge etablieren sich in England mehrere hospizliche Einrichtungen. Bedeutung wird in der Folge vor allem das bereits 1905 im Norden von London eingerichtete St. Joseph's Hospice erlangen, wo Cicely Saunders sieben Jahre lang arbeitete und durch diese Erfahrung massgeblich beeinflusst wurde (Buck 2007).

Cicely Saunders lässt sich zunächst als Pflegende, später auch als Sozialarbeiterin und schliesslich als Ärztin ausbilden. Bereits früh beschäftigt sie sich mit der Schmerzbehandlung von sterbenden Menschen und besucht immer wieder hospizliche Einrichtungen in den USA (Cicely Saundes International 2017), um die dortigen Erfahrungen und Ansätze in ihre Arbeit zu integrieren. Geprägt durch persönliche Erfahrungen in der Begleitung eines unheilbar 
kranken engen Vertrauten gründet Cicely Saunders nach jahrelanger Vorarbeit nach dem Vorbild des Joseph's Hospice 1967 das St. Christopher's Hospice im Süden von London. Es gilt als erstes modernes Hospiz, das die Bereiche der klinischen Versorgung, der Forschung und der Weiterbildung vereint und dies in der Behandlung nutzt. Durch die vielfältige Expertise der Gründerin orientiert sich die Versorgung an den physischen, psychischen, sozialen und spirituellen Bedürfnissen der schwerkranken und sterbenden Menschen. Diesen holistischen Ansatz fügt sie in ihrem Konzept des «total pain» zusammen (Ong/Forbes 2005). Cicely Saunders legt mit ihrer Arbeit die Basis für die weltweite Palliative Care Bewegung, die in den folgenden Jahren einsetzt (Clark/Centeno 2006). Für diese Verdienste wird sie 1980 von der Queen geadelt. Im Alter von 87 Jahren stirbt sie am 14. Juli 2005 im St. Christopher's Hospice (Cicely Saunders International 2017).

Bereits während des Schaffens von Cicely Saunders verlaufen die Entwicklungen in vielen westlichen Ländern parallel. Am Royal Victoria Hospital in Montreal wird 1975 die erste Palliative Care Unit eingerichtet und ein wichtiger Grundstein für die Entwicklung und Etablierung der heutigen palliativen Versorgung gelegt (Hospice Society of the Columbia Valley 2017). In der Schweiz findet eine erste Sensibilisierung 1970 durch die Pflegefachfrau Rosette Poletti in Genf statt, die auf die Bedeutung des palliativen Gedankens aufmerksam macht. Im Nachbarland öffnet 1983 die erste bundesdeutsche Palliative Care Unit in Köln ihre Pforten (Klaschnik/Nauck 1998). Mit dem Ziel, die palliative Versorgung gesamtschweizerisch zu organisieren und voranzutreiben, gründet sich 1988 die Schweizerische Fachgesellschaft für Palliative Medizin, Pflege und Begleitung, die sich heute palliative.ch nennt (palliative.ch o. J.). Im gleichen Jahr formiert sich die European Association for Palliative Care, die heute fast alle europäischen Länder unter einer Dachgesellschaft vereint, richtungsweisende internationale Projekte durchführt und richtungsweisende Empfehlungen formuliert (Clark/Centeno 2006). Sechs Jahre später folgt in Deutschland 1994 die Gründung der Deutschen Gesellschaft für Palliativmedizin (DGP 2014). Bereits 1992 fordert die australische Pharmazeutin und Rechtswissenschaftlerin Margaret Sommerville, dass Palliative Care ein Grundrecht der Menschen darstellt (Annas 1994). Es sollen jedoch noch einige Jahre vergehen, bis der palliative Ansatz international und national deutlich erstarkt und es im zu einer Zunahme entsprechender Angebote im stationären und ambulanten Setting kommt. Deutlichen Aufschwung erhält der Ansatz dann zu Beginn des 21. Jahrhunderts durch eine Anpassung der Definition von Palliative Care der WHO und den Konsequenzen, die sich daraus ergeben.

\section{Notwendigkeit und Verständnis der Palliative Care}

Die Hintergründe der Versorgung von schwerkranken und sterbenden Menschen wurden im vorherigen Abschnitt im Kontext historischer Begebenheiten skizziert. In den folgenden Ausführungen soll sich dem Wesen der Palliative Care weiter genähert und die Notwendigkeit sowie die Grundannahmen dieses Ansatzes beleuchtet werden.

Die Relevanz des palliativen Versorgungsansatzes ergibt sich zunächst durch den epidemiologischen und demografischen Wandel und den damit verbundenen gesellschaftlichen, gesundheits- und sozialpolitischen Herausforderungen. Diese zeigen sich auf unterschiedlichen Ebenen. In den hochentwickelten Nationen führen die Fortschritte im Gesundheitswe- 
sen und die Möglichkeiten der Hightech-Medizin dazu, dass der Tod als unabänderliche Tatsache immer weiter aus dem gesellschaftlichen Bewusstsein verschwindet (Patel/Kruczynski 2015). Auch die steigende Lebenserwartung der Bevölkerung gilt als wichtige Errungenschaft moderner Gesellschaften. Letztgenannter Aspekt hat verschiedene Folgen: Zum einen korreliert das Alter mit dem individuell erhöhten Risiko, eine oder mehrere chronische Krankheiten zu entwickeln, die zwar immer früher diagnostiziert und behandelt, aber nicht geheilt werden können. Dies führt zu einem Anstieg der Lebensjahre, die Betroffene mit einer fortschreitenden Krankheit leben und meist zunehmend alltägliche Einschränkungen bewältigen müssen. Denn häufig sind chronische Krankheiten mit Symptomen assoziiert, die die Lebensqualität der Betroffenen beeinträchtigen und eine nicht mehr auf die Heilung fokussierte Versorgung nötig machen. Die Notwendigkeit einer palliativen bzw. end-of-life fokussierten Versorgung ergibt sich darüber hinaus dadurch, dass aufgrund der Altersstruktur der Bevölkerung die Zahl der sterbenden Menschen prognostisch bis 2050 stark ansteigt wird (BAG/GDK 2010).

Eine erste international verbreitete Definition der Palliative Care wird 1990 von der WHO vorgelegt. Bereits 2002 werden Anpassungen nötig und die WHO konkretisiert und ergänzt ihre Ausführungen. Unter Palliative Care wird heute ein Ansatz verstanden, der die Lebensqualität von unheilbar kranken und sterbenden Menschen und ihrer An- und Zugehörigen ins Zentrum stellt. Die Lebensqualität soll durch das frühzeitige Erkennen sowie die professionelle Einschätzung und Behandlung bestehender körperlicher, psychischer, sozialer und spiritueller Probleme möglichst erhalten und gefördert werden. Der Ansatz zeichnet sich insbesondere dadurch aus, dass er bestehendes Leiden auf ein für Betroffene erträgliches Mass reduziert, das Leben achtet und das Sterben als normalen Prozess des menschlichen Seins respektiert. Dabei soll der Tod weder beschleunigt noch hinausgezögert werden (WHO 2017).

Der Ansatz integriert die verschiedenen Dimensionen des Menschseins und bietet den schwerkranken und sterbenden Menschen die nötige Unterstützung, für ein gutes Leben bis zuletzt. Die Palliative Care wendet sich auch an die jeweiligen An- und Zugehörigen und betrachtet sie als integralen Bestandteil einer Versorgungseinheit. Die Unterstützung reicht dabei über den Tod des schwerkranken Menschen hinaus und begleitet die Hinterbliebenen in ihrer Trauer sowie in ihren psychosozialen Bedürfnissen. Diese Aufgaben werden von einem interprofessionellen Team übernommen, das gemeinsame Ziele verfolgt, die sich an den Bedürfnissen der Betroffenen und deren Umfeld orientieren. Der palliative Ansatz soll dabei frühzeitig in die Behandlung von unheilbar kranken Menschen integriert und mit weiteren Behandlungsoptionen kombiniert werden (WHO 2017).

Eine wichtige Unterscheidung soll in diesem Zusammenhang kurz aufgezeigt werden. Vielfach wird in der Bevölkerung - aber auch von Fachpersonen unterschiedlicher Professionen - die Palliative Care mit der Behandlung, Pflege und Betreuung von sterbenden Menschen gleichgesetzt bzw. der Ansatz darauf reduziert. Palliative Care greift aber deutlich weiter, umfasst eine längere Zeitspanne und soll - im Sinne der oben aufgeführten WHODefinition (2017) - möglichst früh im Behandlungskonzept von fortgeschritten chronisch kranken Menschen berücksichtigt werden. In diesen palliativen Behandlungssituationen ist das Lebensende noch nicht absehbar und mehrere Lebensjahre bei bestmöglicher Lebensqualität sind das Ziel. Damit nimmt die Palliative Care nicht ausschliesslich die sterbenden Men- 
schen in den Blick. Vielmehr wendet sie sich bereits vorausschauend in der gesundheits- und krankheitsbezogenen Grundversorgung an alle Menschen, die an einer unheilbaren, im Verlauf fortschreitenden Krankheit leiden (BAG/GDK 2010). Die palliative medizinische, pflegerische, spirituelle und psychosoziale Behandlung am Lebensende wird als end-of-life care bezeichnet und stellt einen zentralen - aber nicht alleinigen - Teil bzw. den inneren Kreis der Palliative Care dar, in dem die Behandlungsprioritäten überdacht und angepasst werden müssen (Rome et al. 2011).

\section{Palliative Care als Strategie}

Die Aufforderung der WHO, Palliative Care im Sinne der oben präsentierten Definition als integralen Bestandteil der gesundheitlichen Versorgung flächendeckend in die bestehenden Gesundheitssysteme zu implementieren, ist mit verschiedenen Konsequenzen verbunden. Im Vordergrund steht dabei die Organisation, Verbreitung und Verankerung dieses Ansatzes sowohl gesellschaftlich als auch gesundheitspolitisch. Die vielfältigen Herausforderungen, die damit einhergehen, sollen übergeordnet aufgenommen, koordiniert und gemeinsam mit der Politik, den Behörden, den Fachgesellschaften und der Bevölkerung im Rahmen einer Strategie bewältigt werden. Diese Empfehlung wird von der WHO 2002 ausgesprochen (Sepulveda et al. 2002) und in ihrer Resolution von 2014 bekräftigt (WHO 2014).

In der Schweiz werden 2009 die Grundlagen geschaffen, um eine Nationale Strategie Palliative Care gemeinsam mit dem Bund und den Kantonen zu initiieren. Die Strategie erstreckt sich zunächst über sechs Jahre, die in zwei Phasen unterteilt werden. In der ersten Phase, die von 2010-2012 reicht, wird aufgrund einer Bestandsaufnahme die Situation in der Versorgung von schwerkranken und sterbenden Menschen aufgezeigt und der nationale Handlungsbedarf abgeleitet. Als die sechs zentralen Handlungsfelder werden die folgenden Bereiche aufgegriffen: die flächendeckende palliative Versorgung und der Aufbau entsprechender Strukturen, die Finanzierung und der Zugang zu Palliative Care Leistungen für alle Bevölkerungsgruppen, die Sensibilisierung der Bevölkerung, die Aus-, Fort- und Weiterbildung der Health Professionals und der freiwillig Mitarbeitenden, die Etablierung einer Palliative Care fokussierten Forschung sowie allgemein der Schaffung von Rahmenbedingungen für die Einbettung und Umsetzung der Strategie (BAG/GDK 2009). Ein Schwerpunkt in dieser Strategieetappe liegt auf dem Aufbau der spezialisierten Palliative Care, die sich an Menschen mit und in komplexen, sich schnell veränderbaren Gesundheitssituationen bei weit fortgeschrittenen Krankheitsverläufen und deren An- und Zugehörigen richtet. Diese Situationen stellen sich vielfach, aber nicht ausschliesslich, am Lebensende ein. An vielen universitären oder kantonalen Spitälern werden spezialisierte Fachabteilungen eingerichtet und entsprechendes Fachpersonal - zunächst mit dem Fokus auf Pflege und Medizin - weitergebildet. Die Kantone engagieren sich unterschiedlich stark in diesem Bereich, so dass schweizweit grosse Unterschiede in Bezug auf die Unterstützung von Weiterbildungsmassnahmen beobachtet werden können. Die bestehenden ambulanten Angebote der spezialisierten Palliative Care werden in dieser Phase der Strategie angepasst und die Zusammenarbeit zwischen dem stationären und ambulanten Setting ausgebaut und verstärkt. 
Bund und Kantone beschliessen, den begonnenen Weg weiter zu gehen und verlängern 2012 die Nationale Strategie um weitere drei Jahre. Das bis dahin Erreichte wird bilanziert, kritisch evaluiert, Empfehlungen und neue Handlungsfelder werden formuliert. Zu den bis anhin wichtigen Themen wird die Strategie 2013-2015 um folgende Aspekte erweitert: die Förderung der Palliative Care in der Grundversorgung bzw. der Auf- und Ausbau des Palliative Care Angebotes in den Pflegeheimen, im ambulanten Bereich, in den Fachrichtungen Geriatrie und Pädiatrie sowie im Bereich der Versorgung von Menschen mit Behinderungen (BAG/GDK 2012). 2017 wird die Strategie in die «Nationale Plattform Palliative Care» überführt und so das Anliegen einer flächendeckenden Palliative Care Versorgung in der Schweiz weitergeführt. Ziel dieser Plattform ist die weitere Vernetzung und Förderung der Zusammenarbeit der verantwortlichen Akteure sowie der Austausch von Erfahrungs- und Forschungswissen (GDK 2017). Mit der Strategie und der Überführung in neue Gefässe wird den gesellschaftlichen Veränderungen und Bedürfnissen sowie den Aufgaben einer humanistisch geprägten Gemeinschaft, der auch das Wohl der als vulnerabel bezeichneten Gruppe der schwerkranken und sterbenden Menschen ein wichtiges Anliegen ist, Rechnung getragen.

\section{Prinzipien und zentrale Konzepte}

Die Palliative Care fusst auf den Säulen von Autonomie, Ethik und Würde (SAMW 2013), die für die Versorgung von schwerkranken und sterbenden Menschen übergeordnet als handlungsleitend verstanden werden können. Das wichtigste Anliegen des Versorgungsansatzes ist die Erhaltung und Förderung der bestmöglichen Lebensqualität für die von einer lebenslimitierenden, schweren Krankheit betroffenen Menschen. Daneben bestehen weitere zentrale Konzepte, die sich in Anlehnung an die WHO-Definition ergeben und die als Prinzipien der Palliative Care gelten (IAHPC 2017). Die wichtigsten werden im Folgenden kurz dargestellt.

Wie oben bereits im Zusammenhang mit der Entstehung und den Arbeiten von Cicely Saunders dargelegt, stellt der holistische Ansatz ein tragendes Konzept dar. Die mehrperspektivische Betrachtungsweise auf den erkrankten Menschen und sein Umfeld berücksichtigt die verschiedenen Dimensionen des menschlichen Seins und betrachtet sie als physisch, psychisch, sozio-kulturell und spirituelle Einheit unter biografischen Begebenheiten. Des Weiteren sind die Konzepte Individualität, An- und Zugehörige, interprofessionelle Zusammenarbeit und Kommunikation (Baldwin 2011) sowie Symptommanagement, Trauer und deren Begleitung und Spiritualität zentrale Bausteine der Palliative Care.

Die meist mehrdimensional ausgerichteten Behandlungssituationen sollen von den beteiligten Health Professionals gemeinsam und aus der subjektiven Sicht der betroffenen Menschen erfasst werden. Daher steht zu Beginn einer palliativen Behandlungs- und Pflegebeziehung die sorgfältige Erhebung und Einschätzung der Bedürfnisse bzw. der möglichen körperlichen und psychischen Symptome. Dazu wird zunächst ein medizin- und pflegeorientiertes Assessment durchgeführt, in dessen Zentrum der erkrankte Mensch und seine An- und Zugehörigen stehen. Daneben werden soziale und spirituelle Aspekte erfasst (DKG/AWMF 2015). Gemeinsam mit den Patientinnen, Bewohnerinnen oder Klienten werden in einem nächsten Schritt in Abhängigkeit mit dem Krankheitsstadium die Ziele der palliativen Behandlung festgelegt und entsprechende Interventionen geplant und durchgeführt. 
Für eine schnelle und dennoch strukturierte Erfassung der aktuellen Situation von schwerkranken und sterbenden Menschen hat sich neben den pflegewissenschaftlichen Grundlagen professionellen Handels, wie beispielsweise dem Pflegeprozess (Brobst/Georg 2007), das SENS-Modell bewährt (Eychmüller 2012). Die Buchstaben der Abkürzung stehen für die Bereiche Symptommanagement, Entscheidungsfindung, Netzwerk sowie Support für Angehörige und bilden übersichtlich die zentralen Punkte einer palliativen Versorgung ab. Als wichtiges Prinzip gilt, dass die durchgeführten Interventionen regelmässig im Hinblick auf ihren Erfolg evaluiert und wenn nötig in Absprache mit den Betroffenen und deren Umfeld angepasst werden. Um die Wirksamkeit der Massnahmen in Bezug auf die Lebensqualität der betroffenen Patienten, Bewohnerinnen oder Klienten zu erfassen, hat sich die Palliative Outcome Scale (POS) als hilfreich erwiesen (Rugno/De Carlo 2016).

\section{Herausforderungen}

Die Palliative Care ist heute in vielen Ländern integraler Bestandteil des Gesundheitswesens und wird in Europa und weltweit umgesetzt (Reville/Foxwell 2014). Dennoch bestehen in verschiedenen Bereichen Herausforderungen, denen sich die Gesellschaft und die an der Umsetzung des Ansatzes beteiligten Akteure auf unterschiedlichen Ebenen stellen müssen. Im Folgenden sollen die wichtigsten Aspekte in diesem Zusammenhang dargestellt werden.

In den Anfängen der Palliative Care lag der Schwerpunkt in der Versorgung von Menschen mit Tumorerkrankungen. Durch die epidemiologische Häufigkeit und die oft mit einer unmittelbar lebensbedrohlichen Krankheit assoziierten Behandlungssituationen in der Onkologie wurden viele Konzepte und Behandlungsrichtlinien vor diesem Hintergrund entwickelt. Auch heute behandeln viele Fach- und Forschungspublikationen die Palliative Care unter onkologischen Gesichtspunkten. Inzwischen richtet sich die palliative Versorgung aber nicht mehr ausschliesslich an Menschen mit Tumorerkrankungen, auch wenn ein grosser Teil der Patientenklientel noch immer aus diesem Bereich stammt. Durch die konsequente Orientierung an der WHO-Definition wird immer mehr Menschen mit chronischen Erkrankungen, wie zum Beispiel fortschreitende Herz- und/oder Niereninsuffizienz oder Multiple Sklerose, Morbus Parkinson oder Demenz die Palliative Care zuteil. So hat in den vergangenen Jahren eine Erweiterung der palliativen Angebote in diesem Bereich stattgefunden (WHO 2015), die zukünftig weiter ausgebaut und an den Bedürfnissen dieser Zielgruppe ausgerichtet werden muss. Dies gilt auch für die Fachbereiche Geriatrie und Pädiatrie. Den Bedürfnissen von alten Menschen und Kindern muss auch in der palliativen Versorgung in besonderer Art und Weise Rechnung getragen werden. Die grosse Bedeutung einer geriatrischen Palliative Care leitet sich zum einen aus den demografischen Prognosen und zum anderen vom bereits erwähnten erhöhten Erkrankungsrisiko im Alter ab (WHO 2011). In diesem Zusammenhang soll die Herausforderung Palliative Care bei Menschen mit Demenz nicht unerwähnt bleiben. Die Personengruppe hat durch die Besonderheiten der zugrundeliegenden Krankheit besondere Bedürfnisse, denen bis ans Lebensende mit entsprechenden Konzepten begegnet werden muss (Harris 2007). Auf dem Gegenpol des Lebenskontinuums konnte die Schweizerische PELICAN-Studie den Bedarf an einer pädiatrisch-orientierten Palliative Care aufzeigen (Bergsträsser et al. 2016). Die Etablierung entsprechender Angebote wird zu einer weiteren Differenzie- 
rung in den palliativspezifischen Ausrichtungen der genannten Bereiche - vornehmlich in der Medizin und in der Pflege - führen.

Eine weitere Herausforderung zeigt sich bei der frühen Integration der Palliative Care in einen geplanten und/oder begonnenen Behandlungsprozess. Eine frühe Berücksichtigung und Umsetzung der palliativen Gesichtspunkte und Behandlungsoptionen verlängert die Lebenserwartung der schwer erkrankten Menschen bei besserer Lebensqualität, reduziert die Belastungen von pflegenden Angehörigen und trägt dazu bei, dass Kosten gesenkt werden (Groh et al. 2013). Die frühe Berücksichtigung dieses Ansatzes und die Kombination mit bestehenden Behandlungselementen wird daher von der WHO (2017) und verschiedenen Fachgesellschaften empfohlen (Ferrell et al. 2017). Dennoch wird das spezialisierte palliative Versorgungsangebot häufig erst sehr spät genutzt und die Patienten an entsprechende Facheinrichtungen überwiesen. Dies führt vielfach dazu, dass bestehende Symptome nur unzureichend gelindert werden und somit unnötiges Leiden verursacht wird. Als Gründe dafür werden unterschiedliche Einschätzung der verantwortlichen Fachpersonen über Schwere und Verlauf der Krankheit und somit der Notwendigkeit palliativer Leistungen aufgeführt (Fink 2015). Das falsche Verständnis, dass ein palliativer Behandlungsansatz das Leben der schwerkranken Menschen vorzeitigt beenden könnte und unzureichende Kommunikationsstrukturen zwischen den Health Professionals, den Betroffenen sowie deren Familien zählen zu den weiteren Gründen, warum gegen oder erst sehr spät für die Option der Palliative Care entschieden wird (Morita et al. 2005).

Herausforderungen liegen auch im interprofessionellen Ansatz der Palliative Care selbst. Die Beteiligung verschiedener Professionen in unterschiedlichen Phasen der palliativen Behandlung machen eine sorgfältige fachübergreifende Kommunikation und eine wertschätzende und vertrauensvolle Zusammenarbeit nötig. Dabei erweist sich die interprofessionelle Kommunikation nicht einfach nur als Austausch zwischen mehreren Beteiligten, sondern muss vor dem Hintergrund der Logiken der verschiedenen Professionen und der soziokulturellen Begebenheiten betrachtet werden. Die häufigsten Gründe für eine nicht gelingende interprofessionelle Kommunikation sind starre Verwaltungs- und Hierarchiestrukturen sowie die nicht gemeinsam festgelegten, sondern vielmehr nebeneinander stehenden Ziele und durchgeführten Interventionen im Behandlungsprozess (Hartrick Doane et al. 2012). Dies hat auch Auswirkungen auf die Zusammenarbeit zwischen den einzelnen Akteuren des Palliative Care Teams. Als grosse Barriere erweisen sich aus Sicht der Patienten, der An- und Zugehörigen und der Health Professionals selbst die oft unklaren Rollen und Erwartungen der beteiligten Personen. Die bereits erwähnten bestehenden Unsicherheiten in Bezug auf den Krankheitsverlauf und der Mangel an Zusammenarbeit verhindern jedoch eine effektive patientenorientierte Versorgung (Oishi/Murtagh 2014).

Die Zusammensetzung der Palliative Care Teams mit Personen unterschiedlicher Professionen muss ebenfalls vor dem Hintergrund der Kompetenzen und Zuständigkeiten betrachtet werden. Palliative Care beinhaltet Aspekte der Grundversorgung wie auch der Spezialisierung und deren Unterscheidung ist nicht immer klar. In nicht wenigen Situationen der palliativen Versorgung sind Teilaspekte beider Bereiche enthalten, beispielsweise dann, wenn die körperlichen Symptome auf ein erträgliches Mass reduziert werden konnten, sich jedoch das familiäre Umfeld als äussert instabil und fragil darstellt. Dies führt nicht nur zu Unsicherhei- 
ten in den Zuständigkeiten bzw. in Bezug auf die beste Versorgungsoption, sondern auch im Hinblick auf die Bedeutung der professionellen Kompetenzen innerhalb der Teams. Die Fragen der Kompetenzen haben darüber hinaus Auswirkungen auf die Aus-, Fort- und Weiterbildungsangebote im Bereich der Palliative Care. Als erste Orientierungshilfe können hier die Empfehlungen der European Association of Palliative Care (Gamondi/Larkin/Payne 2013a, 2013b) herangezogen werden, die zehn Kernkompetenzen für Health Professionals in der Palliative Care formulieren. Die Herausforderung für die Teams der Palliative Care in der praktischen Umsetzung besteht jedoch darin, die für die aktuelle Situation bzw. für das aktuelle Problem und die Durchführung geeigneter Massnahmen die am besten geeignete Berufsgruppe zu bestimmen sowie ihr im Anschluss daran die temporäre Federführung und die Handlungsumsetzung zu übertragen.

Eine übergeordnete Herausforderung, die alle bisherigen Aspekte umfasst, stellt die Finanzierung der palliativen Versorgung dar. Palliative Care ist ein weitgehend akzeptiertes Versorgungskonzept, bisher jedoch kein Begriff in den Ausführungen zur obligatorischen Krankenpflegeversicherung. Dies führt dazu, dass zwar die Kosten für die Behandlung einer Krankheit und die benötigte Pflege dort abgedeckt und somit übernommen werden, dies jedoch nicht für den Bereich der psychosozialen, spirituellen und psychologischen Unterstützung gilt (BAG/GDK 2013). Aufgrund dieser Begebenheiten kann der holistische Ansatz der Palliative Care nicht umgesetzt und ein wichtiges Anliegen dieser Versorgung nicht gewährleistet werden. Die Fragen der Finanzierung stellen sich darüber hinaus auch im Hinblick auf die Bereitstellung ausreichender Personalressourcen und auf die Weiterbildung der Health Professionals. Denn den vielfältigen und komplexen Herausforderungen der Palliative Care kann nur mit der umfangreichen Expertise von gut aus- und weitergebildeten Fachpersonen in Ergänzung zu den freiwillig Mitarbeitenden begegnet werden. Um ein reibungsloses Miteinander aller am Prozess Beteiligten im Sinne einer personen- und bedürfnisorientierten Versorgung zu gewährleisten, müssen Rahmenbedingungen geschaffen werden, in denen dieses Anliegen realistisch umgesetzt werden kann.

\section{Fazit und Ausblick}

Der Ansatz der palliativen Versorgung ist vor allem in den westlichen Industrienationen fester Bestandteil vieler Gesundheitssysteme. Viele dieser Länder - vornehmlich die Schweiz und Deutschland - haben den Aufbau und die Etablierung der Palliative Care strukturiert anhand einer Strategie begleitet und somit gesellschaftlich und gesundheitspolitisch verankert. Die Versorgungsstrukturen sowohl in der grundversorgenden als auch in der spezialisierten Palliative Care konnten im Rahmen dieses Vorgehens in vielen Bereichen ausgebaut werden.

Die konsequente Orientierung an den Bedürfnissen der schwerkranken und sterbenden Menschen, mit dem Ziel einer bestmöglichen Lebensqualität bis zuletzt, zeichnet den palliativen Ansatz als humanes Behandlungskonzept aus. Es ist von Vorteil, die vielfältigen Aufgaben und die mehrperspektivischen Herausforderungen im interprofessionellen Team zu bewältigen. Eben diese Merkmale sind verbunden mit Herausforderungen, die die Gesellschaft und mit ihr insbesondere das Gesundheits- und Sozialwesen in den nächsten Jahren herausfordern werden. Um dem gesellschaftlichen Auftrag einer guten und den Qualitätskriterien entspre- 
chenden Palliative Care gerecht zu werden, müssen die benötigten finanziellen und personellen Ressourcen in ausreichendem Mass zur Verfügung stehen. Nur dann können die bisherigen Errungenschaften einer an den Prinzipien der Ethik, Autonomie und Würde orientierten palliativen Versorgung, die die individuellen Bedürfnisse der schwerkranken und sterbenden Menschen berücksichtigt, langfristig sichergestellt werden.

Vor dem Hintergrund des globalen und lokalen Wandels der Gesellschaften und den damit verbundenen neuen Aufgaben und Herausforderungen muss das auf die Lebensqualität ausgerichtete Versorgungskonzept allen bedürftigen Menschen zugänglich gemacht und die bestehenden Barrieren ausgeräumt und behoben werden. Die Sensibilisierung der Bevölkerung und der gesundheitsorientierten Fachpersonen muss aufrechterhalten und fortgesetzt werden. So gewinnt die Palliative Care weiter an Bedeutung und es entstehen Strukturen, die möglichst für alle ein gutes Leben bis zuletzt ermöglichen.

\section{Literaturverzeichnis}

Annas, George J. (1994). Margret Somerville. A Refreshing Challange. Journal of Contemporary Health \& Law, 11 (1), Article 4, Zugriff am 29.03.2017 auf http://scholarship.law.edu/jchlp/vol11/iss1/4.

Baldwin, Moyra A. (2011). Attributes of palliative caring. In: Moyra A. Baldwin und Jan Woodhouse (Hg.). Key concepts in Palliative Care (7-11). London: Sage Publication.

Bergsträsser, Eva, Eva Cignacco, Karin Zimmermann, Katri Eskola, Katrin Marfurt-Russenberger, Patricia Luck, Anne-Sylvie Ramelet, Patricia Fahrni-Nater und Anouk Dorsaz (2016). Wie Kinder in der Schweiz sterben. Schlüsselergebnisse der PELICAN-Studie. Zugriff am 29.03.2017 auf http://www.kispi.uzh.ch/fzk/de/abteilungen/uebersicht/ppc/Documents/Pelican_dd.pdf.

Brobst, Ruth A. und Jürgen Georg (2007). Der Pflegeprozess in der Praxis. 2. Auflage. Bern: Huber Verlag.

Buck, Joy (2007). Reweaving a Tapestry of Care: Religion, Nursing, and the Meaning of Hospice, 1948-1978. Nursing History Review, 15, 113-145.

Bundesamt für Gesundheit (BAG) und Schweizerische Konferenz der Gesundheitsdirektorinnen und direktoren (GDK) (Hg.) (2013). Finanzierung der Palliative-Care-Leistungen der Grundversorgung und der spezialisierten Palliative Care (ambulante Pflege und Langzeitpflege). Internet-Seite. Zugriff am 29.03.2017 auf https://www.bag.admin.ch/bag/de/home/themen/strategien-politik/nationalegesundheitsstrategien/strategie-palliative-care/versorgung-und-finanzierung-in-palliative-care/fin anzierung-der-palliative-care.html.

Bundesamt für Gesundheit (BAG) und Schweizerische Konferenz der Gesundheitsdirektorinnen und direktoren (GDK) (Hg.) (2012). Nationale Strategie Palliative Care 2013-2015. Zugriff am 15.03.2017 auf https://www.bag.admin.ch/dam/bag/de/dokumente/nat-gesundheitsstrategien/st rategie-palliative-care/nationale-strategie.pdf.download.pdf/07_D_Nationale_Strategie_Palliative_ Care_2013-2015.pdf. 
Bundesamt für Gesundheit (BAG) und Schweizerische Konferenz der Gesundheitsdirektorinnen und direktoren (GDK) (Hg.) (2010). Nationale Leitlinien Palliative Care. Zugriff am 15.03.2017 auf https://www.bundespublikationen.admin.ch/cshop_mimes_bbl/8C/8CDCD4590EE41ED6B5FCE8 3EDD643430.pdf.

Bundesamt für Gesundheit (BAG) und Schweizerische Konferenz der Gesundheitsdirektorinnen und direktoren (GDK) (Hg.) (2009). Nationale Strategie Palliative Care 2010-2012. Zugriff am 15.03.2017 auf https://www.bag.admin.ch/dam/bag/de/dokumente/nat-gesundheitsstrategien/str ategie-palliative-care/nationale-strategie-palliative-care-2010-2012.pdf.download.pdf/10_D_Natio nale_Strategie_Palliative_Care_2010-2012.pdf.

Cicely Saunders International (2017). Dame Cicely Saunders Biography. Internet-Seite. Zugriff am 21.03.2017 auf

http://cicelysaundersinternational.org/dame-cicely-saunders/st-christophers-hospice.

Clark, David und Carlos Centeno (2006). Palliative Care in Europe: an emerging approach to comparative analysis. Clinical Medicine, 6, 197-201.

Deutsche Krebshilfe (DKG) und Arbeitsgemeinschaft der Wissenschaftlichen Medizinischen Fachgesellschaften (AWMF) (2015). S3-Leitlinie Palliativmedizin für Patienten mit einer nicht heilbaren Krebserkrankung. Internet-Seite. Zugriff am 15.03.2017 auf http://www.awmf.org/uploads /tx_szleitlinien/128-001OLl_S3_Palliativmedizin_2015-07.pdf.

DGP Deutsche Gesellschaft für Palliativmedizin (2014). Die DGP - eine lebendige Gesellschaft. Internet-Seite. Zugriff am 19.03.2017 auf www.dgpalliativmedizin.de.

Eychmüller, Steffen (2012). SENS macht Sinn - Der Weg zu einer neuen Assessmentstruktur in der Palliative Care. Therapeutische Umschau, 69, 87-90.

Ferrell, Betty R., Jennifer S. Temel, Sarah Temin, Erin R. Alesi, Tracy A. Balboni, Ethan M. Basch, Janice I. Firn, Judith A. Paice, Jeffrey M. Peppercorn, Tanyanika Phillips, Ellen L. Stovall, Camilla Zimmermann und Thomas J. Smith (2017). Integration of Palliative Care into Standard Oncology Care: American Society of Clinical Oncology Clinical Practice Guideline update. Journal of Clinical Oncology, 35 (1), 96-114.

Fink, Regina M. (2015). Review of a study on late referral to a palliative care consultation service: Length of stay and in-hospital mortality outcomes. Journal of the Advanced Practitioner in Oncolo$g y, 6,597-610$.

Gamondi, Claudia, Philip Larkin und Sheila Payne (2013a). Core competencies in palliative care: an EAPC White Paper on palliative care education - part 1. European Journal of Palliative Care, 20(2), 86-91.

Gamondi, Claudia, Philip Larkin und Sheila Payne (2013b). Core competencies in palliative care: an EAPC White Paper on palliative care education - part 2. European Journal of Palliative Care, 20(2), $140-144$. 
GDK Schweizerische Konferenz der Gesundheitsdirektorinnen und -direktoren (2017). Nationale Plattform Palliative Care. Internet-Seite. Zugriff am 20.02.2017 auf http://www.gdk-cds.ch/index.php.

Groh, Gesa, Birgit Vyhnalek, Berend Feddersen, Monika Führer und Gian D. Borasio (2013). Effectiveness of a specialized outpatient palliative care service as experienced by patients and caregivers. Journal of Palliative Medicine, 16(8), 848-856.

Harris, Dylan (2007). Forget me not: Palliative Care for people with dementia. Postgraduate Medical Journal, 83, 362-366.

Hartrick Doane, Gweneth, Kelli Stajduhar, Elizabeth Causton, Darcee Bidgood und Amy Cox (2012). End-of-Life Care and Interprofessional Communication. Not simply a Matter of „more“. Health and Interprofessional Practice, 1(3), 1028-1042.

Hospice Society of the Columbia Valley (2017). Canadian Hospice Palliative Care Association. Internet-Seite. Zugriff am 15.03.2017 auf http://www.hospicesocietycv.com/canadian/.

International Association of Hospice and Palliative Care IAHPC (2017). Principles of Palliative Care. Internet-Seite. Zugriff am 15.03.2017 auf https://hospicecare.com/about-iahpc/publications /manuals-guidelines-books/getting-started/6-principles-of-palliative-care.

Klaschnik, Eberhard und Friedemann Nauck (1998). Historische Entwicklung der Palliativmedizin. Zentralblatt für Chirurgie, 123(6), 620-623.

Morita, Tatsuya, Tatsuo Akechi, Masayuki Ikenaga, Yoshiyuki Kizawa, Hiroyuki Kohara, Taketo Mukaiyama, Toshimichi Nakaho, Nobuaki Nakashima, Yasuo Shima, Tatsuhiro Matsubara, und Yosuke Uchitomi (2005). Late referral to specialized palliative care services in Japan. Journal of Clinical Oncology, 23(12), 2637-2644.

Oishi, Ai und Fliss EM Murtagh (2014). The challenges of uncertainty and interprofessional collaboration in palliative care for non-cancer patients in the community: A systematic review form patients, carers and health-care professionals. Palliative Medicine, 28(9), 1081-1098.

Ong, Chi-Keong und Duncan Forbes (2005). Embracing Cicely Saunder's concept of total pain, BMJ, $331,576-577$.

palliative.ch - Schweizerische Gesellschaft für Palliative Medizin, Pflege und Begleitung. Die Geschichte der Palliative Care. Internet-Seite. Zugriff am 15.03.2017 auf https://www.palliative.ch/de/palliative-care/die-geschichte-der-palliative-care/.

Patel, Kashyap und Mary Kruczynski (2015). Palliative Care and end-of-life Care: Issues, Challanges, and possible Solution in the United States. The American Journal of Managed Care, 21(6), 195196.

Reville, Barbara und Anessa M. Foxwell (2014). The global state of palliative care - progress and challenges in cancer care. Annals of Palliative Medicine APM, 3(3), 129-138.

Rome, Robin B., Hillary H. Luminais, Deborah A. Bourgeois und Christopher M. Blais (2011). The Role of Palliative Care at the End of Life. The Ochsner Journal, 11, 348-352. 
Rugno, Fernanda C. und Marysia M. Rodrigues do Prado De Carlo (2016). The Palliative Outcome Scale (POS) applied to clinical practice and research: an integrative review. Rev. Latino-Am. Enfermagem, 24, 2764-2774.

Schweizer Akademie der Medizinischen Wissenschaften SAMW (2013). Palliative Care. Medizinethische Richtlinien und Empfehlungen. Internet-Seite. Zugriff am 15.03.2017 auf http://www.samw.ch/de/Publikationen/Richtlinien.html.

Saunders, Cicely (2001). The Evolution of Palliative Care. Journal of the Royal Society of Medicine, 94, 430-432.

Sepulveda, Cecilia, Amanda Marlin, Tokuo Yoshida und Andreas Ullrich (2002). Palliative Care: The World Health Organization's Global Perspective. Journal of Pain and Symtom Managment, 24(2), 91-96.

Student, Johann C., Albert Mühlum und Ute Student (2016). Soziale Arbeit in Hospiz und Palliative Care. 3. Auflage. München: Ernst Reinhardt Verlag.

Twycross, Robert G. (2003). Introducing Palliative Care. 4. Edition. Abingdon: Radcliffe Medical Press.

WHO (2017). Definition of Palliative Care. Internet-Seite. Zugriff am 15.03.2017 auf http://www.who.int/cancer/palliative/definition/en/.

WHO (2015). Palliative Care for non-communicable diseases. A global snapshot in 2015. Internet-Seite. Zugriff am 17.03.2017 auf http://www.who.int/ncds/management/palliative-care/palliative-careNCDs/en/.

WHO (2014). Strengthening of Palliative Care as a Component of Integrated Treatment throughout the Life Course. Journal of Pain \& Palliative Care Pharmacotherapy, 28(2), 130-134.

WHO (2011). Palliative Care for older people: better practice. Internet-Seite. Zugriff am 20.03.2017 auf www.euro.who.int. 